\title{
Articles
}

The Person and the Challenges

Volume 7 (2017) Number 1, p. 7-36

DOI: http://dx.doi.org/10.15633/pch.1978

Borislav Dadić

University of Zadar, Croatia

\section{Principio di Individiuazione nella Costituzione della Persona Umana}

\section{The Principle of Individuation in the Constitution of the Human Person}

\begin{abstract}
It is generally considered that the principle of individuation is matter and that this doctrine comes from Aristotle. This paper will examine how medieval philosophers approached this problem and we will show that they offered various theories by which the principle of individuation could be matter, form, combination of matter and form, accidents or some special principle such as haecceitas, which we find in the school of J. Duns Scotus. We will assess how all these theories resolve the problem only partially, and thus indicate the need of a single principle of individuation. This becomes particularly relevant in the context of the metaphysical study of a human person. Our research will show that it is actus essendi, which is not only the act of all acts, but also the principle of all principles.
\end{abstract}

\section{Keywords}

principle of individuation, Thomas Aquinas, John Duns Scotus, haecceitas, actus essendi, human person. 


\section{Introduzione}

Occorre notare che il principio di individuazione ha suscitato da sempre l'interesse degli studiosi e questa cosa la possiamo notare anche nei nostri tempi. Alcuni autori gli davano un significato importante. Così, per esempio, H. Heimsoeth lo colloca tra i problemi più importanti del pensiero metafisico Occidentale. ${ }^{1}$ Quando domandiamo che cosa rende un essere appartenente a una specie, oppure cosa rende un ente diverso dall'altro, la maggioranza sarà d'accordo che si tratta della forma, poiché essa definisce la specie e definisce anche cosa sarà un ente ed è quindi essa che da l'essenza all'ente.

Tuttavia, se domandiamo cosa rende un ente esattamente tale ovvero cosa lo rende diverso dall'altro ente della stesse specie non troviamo più la concordia sulla risposta. Anzi, le risposte che vengono date sono piuttosto diverse. Il problema dell'individuazione dellente, ovvero cosa rende il Socrate esattamente tale individuo e non il Platone o qualcun altro, nasconde, oltre il problema dell'individualità, anche il problema dell'identità e dell'unicità dellente. I filosofi medievali erano ben consapevoli di questo fatto. Difatti, loro consideravano l'individualità primariamente come un'unità numerica. ${ }^{2}$ D’altro canto, la maggioranza sarà d’accordo che per una soluzione adeguata del problema dell'individuazione di una persona umana sarà necessario enumerare alcune caratteristiche specifiche che essa possiede e che potrebbero spiegare la sua individualità e unicità. Tuttavia, la domanda è se questo raggiunge la profondità dell'ultimo principio di individuazione, il principio che mi rende una persona diversa di tutte le altre? Cercheremo di risponderlo in questo lavoro.

\section{Il principio di individuazione secondo Platone e Aristotele}

Il noto studioso italiano del pensiero antico, G. Reale, sottolinea che il problema del principio dell'individuazione non si è posto neanche nel pensiero antico e che tale cosa avviene solamente nel pensiero filosofico medievale. Anche se si ritiene che Aristotele insegnava che il principio di individuazione è la materia,

${ }^{1}$ Cf. R. Meßner, Il principio di individuazione nella visione scotista, „Idee” 64 (2007), p. 19.

${ }^{2}$ Cf. P. King, The Problem of Individuation in the Middle Ages, „Theoria” 66 (2000), p. 159184; viene citata una e-variante sul'indirizzo: http://individual.utoronto.ca/pking/articles/ Mediaeval_Individuation.pdf (07.12.2013). 
va osservato che ci sono nelle sue opere le parti che giustificano tale ipotesi, ma anche quelle che sostengono la tesi che il principio di individuazione è la forma. ${ }^{3}$

È noto che Platone divideva la realtà in realtà vera, celeste, nella quale egli pone il suo mondo delle Idee, e in realtà sublunare, che ha un certo tipo di realtà, ma non quella vera; anzi, è una pallida imitazione del mondo delle Idee. L> unica vera realtà sono le Idee. ${ }^{4}$ Ogni singola Idea, in qualche modo, se il modello al Demiurgo, rappresenta quello che nel mondo sublunare sarà una coppia di un tipo di ente. In questo concetto della realtà il problema dell'individuazione non ha senso per cui possiamo concordare con la proposizione di G. Reale sopracitata.

Aristotele ha rifiutato al mondo delle Idee di Platone e ha affermato questo mondo come la vera realtà. Secondo lui questo mondo si trova nella parte sublunare, dove valgono le leggi della generazione, della mutazione e del corruzione. Ciò nonostante, è la vera realtà e questo proprio grazie alla forma.

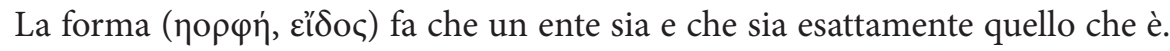
Tuttavia, anche la forma è, come era il caso delle idee di Platone (ì $\delta \dot{\varepsilon} \alpha, \varepsilon \varepsilon^{\prime} \delta \circ \varsigma$ ), uguale per un'intera specie. Ė vero che ogni singolo ente ha una sua forma, ma essa non è diversa dalla forma di un altro ente della stessa specie. Per cui, affinché un ente sia uno numericamente, sarà necessario che lo faccia un altro principio diverso dal quello della forma. Sembrerebbe che per Aristotele quel principio era la materia $(\dot{v} \lambda \eta)$. Infatti, affinché qualcosa sia individuale, cioè indivisibile, deve essere uno. E l'uno per Aristotele è "la misura di tutte le cose, perché conosciamo i costitutivi di una cosa quando la dividiamo o secondo la quantità o secondo la forma. E l'uno è indivisibile per questa ragione: perché in ogni genere di cose ciò che è primo è indivisibile".

${ }^{3}$ Cf. G. Reale, Individuo, in: G. Reale, Storia della filosofia antica, V: Lesico indici e bibliografia, Milano 1997, ed. Vita e Pensiero, p. 142.

${ }^{4}$ Questa è la concezione della realtà che troviamo nelle opere di Platone che sono state preservate fino ai nostri giorni. È vero che molti studiosi del pensiero di Platone di oggi, guidati da Giovanni Reale, considerano un'altra ipotesi, conosciuta sotto il nome delle „dottrine non scritte” di Platone (cf. G. Reale, Per una nuova interpretazione di Platone. Rilettura della metafisica dei grandi dialoghi alla luce della „Dottrine non scritte”, Milano 1993, ed. Vita e Pensiero). In questo paragrafo le Idee sono scritte con una lettera maiuscola perché rappresentano enti eterni, autonomi, spirituali e immutabili e sono diverse dalle idee nella nostra mente, che esistono solamente come degli enti della mente.

${ }^{5}$ Aristotele, Metafisica, 1053 a 18-22. Quando Aristotele in questo punto parla della divisione secondo la qualità e forma, questo „secondo la forma” bisogna considerare come „secondo la qualità". 
Coloro che considerando che per Aristotele il principio di individuazione è la materia espongono diversi pezzi della sua opera, però il pezzo cruciale è quando in Metafisica discute la relazione tra la forma e la generazione di un nuovo ente dove dice che alla fine avviene „la realizzazione di una forma di una data specie realizzata in queste carni ed ossa: per esempio Callia e Socrate; e, questi, sono diversi per la materia (essa è, infetti, diversa nei diversi individui), ma sono identici per la forma (la forma, infatti, è indivisibile)". ${ }^{6}$

E. Regis considera questo punto rilevante per la discussione sul principio di individuazione, anzi lo considera l'argomento più chiaro per la tesi che la materia è il principio di individuazione. Tuttavia, la sua critica principale è che la materia, in quanto potenza pura, manca la parte più propria dell'essere individuale, cioè la quiddità, ${ }^{7}$ per cui non gli è chiaro come la materia potrebbe essere lorigine della quiddità per una sostanza complessa particolare e sensibile. Poiché, infatti, noi generalmente diciamo che una sostanza particolare sia 'questo' o 'quello'.

Per analizzare il problema in fondo, nella sua ampiezza e profondità, bisogna ricordare la nozione aristotelica della sostanza. Infatti, per lui essa è a volte principalmente il synolon, qualcosa di concreto e individuale ( $\tau$ ó $\delta \varepsilon \tau \iota)$, ma talora viene intesa anche come la forma. In questo testo vediamo che dice che „il sinolo e la forma sono due differenti significati della sostanza: il sinolo è la sostanza costituita dall'unione della forma con la materia, l'altra è la sostanza nel senso di forma in quanto tale. Tutte le sostanze intese nel primo significato sono soggette a corruzione, così come sono soggette a generazione. Invece, la forma non è soggetta a corruzione e neppure a generazione: infatti, non si genera lessenza di casa, ma solo l'essere di questa casa qui; le forme esistono o non esistono senza che di esse vi sia processo di generazione e di corruzione: è chiaro, infatti, che nessuno le genera né le produce," e poi Aristotele spiega perché per una sostanza individuale non si può raggiungere una definizione adeguata e che la ragione per questo è la materia, "per questa ragione, delle sostanze sensibili particolari non cè né definizione né dimostrazione, in quanto hanno materia, la cui natura implica la possibilità di essere e di non essere: perciò tutte queste sostanze sensibili individuali sono corruttibili".

${ }^{6}$ Aristotele, Metafisica, 1034 a 5-8. Altri punti di Metafisica che spesso vengono riportati a favore della tesi che la materia è il principio di individuazione sono questi: 1016 b 32; $1035 \mathrm{~b}$ 27-31; 1054 a 34; 1074 a 31-34, così come un punto dellopera De caelo, 270 a 6-b3.

7 Cf. E. Regis, Aristotle's 'principle of individuation', „Phronesis” 21 (1976), p. 160. E. Regis usa l'espressione "thisness", che ricorda chiaramente il concetto haecceitas di Duns Scoto.

${ }^{8}$ Aristotele, Metafisica, 1039 b 20-30. 
Un punto molto indicativo, che viene spesso citato a favore della tesi che la materia è il principio di individuazione (Metafisica, 1035 b 27-31), è quello in cui Aristotele dice che quando parliamo di un uomo concreto, per esempio Socrate, e non dell'uomo in generale, allora esso possiede una materia non generale, ma

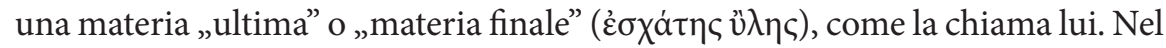
suo commento di questo punto, Tommaso d'Aquino fa un passo avanti spiegando che qui l'individuo indica una cosa composta dalla "materia finale", che lui, più direttamente, chiama anche la materia individuale. ${ }^{9}$ Sembra chiaro che entrambi pensano che è la materia quella che dell'uomo, inteso generalmente, fa un individuo concreto, ovvero Socrate nellesempio di Aristotele. Tuttavia, non possiamo non notare che entrambi parlano di "una materia concreta", e non semplicemente della materia come tale. Si pone subito la domanda: Che cosè, o meglio ancora quale è il principio, che fa che la materia sia esattamente "quella materia concreta", ovvero "finale" come viene chiamata da Aristotele, e "individuale" da Tommaso? E quale è il ruolo del tale principio nel processo dell'individuazione di un ente?

Sono proprio queste domande che hanno spinto alcuni studiosi a cercare, nel pensiero di Aristotele, degli argomenti a favore della tesi che la forma sia questo principio di individuazione dellente. Coloro che pensavano che si trattasse effettivamente della forma portavano come l'argomento più forte un punto di Metafisica di Aristotele dove lui dice che „delle cose che non rientrano nello stesso genere (dei colori, dei suoni, delle sostanze, delle qualità) le cause saranno diverse, eccetto che per analogia; e anche delle cose che rientrano sotto la stesa specie le cause saranno diverse, non specificamente diverse, ma numericamente diverse nei diversi individui: la tua materia, la tua forma e la tua causa efficiente numericamente non sono identiche alle mie, mentre sono universalmente e specificamente identiche". ${ }^{10}$

Abbiamo già parlato dell'importanza dell'unità numerica dell'essere, che lo rende unico rispetto alle altre creature della stessa specie. Questa teoria dell'individuazione mette in risalto il fatto che l'unicità dell'ente deriva dall'unicità della sostanza e dell'essenza e che questi derivano dall'unica forma sostanziale dell'ente. $^{11}$

${ }^{9}$ Cf. Tommaso d'Acquino, Sententia super Metaphysicam, lib. 7, lec. 10., n. 1490: „Sed singulare dicit aliquid compositum 'ex ultima materia', idest materia individuali."

${ }^{10}$ Cf. Aristotele, Metafisica, 1071 a 25-29. Altri punti di Metafisica che spesso vengono riportati a favore della tesi che la materia è il principio di individuazione sono questi: $1038 \mathrm{~b}$ 14-15; 1041 b 7-8, così come un punto dell'opera De anima: 412 a 8-9; 407 b23-24.

${ }^{11}$ Cf. Aristotele, Metafisica, 1038 b 14-15. 
Un'ulteriore argomento per questa tesi si trova nellopera De anima di Aristotele, dove lui analizza quali sono i possibili significati con i quali possiamo chiamare una cosa sostanza. Aristotele dice che la sostanza è nel primo senso la materia, che di per sé non è determinata; nel secondo senso è la forma, grazie alla quale la materia viene chiamata come questa cosa concreta; e nel terzo senso è una unione di materia e di forma. ${ }^{12}$ Da questo è evidente che la materia di un ente sensibile può essere indicata come 'questo qualcosa' solo grazie alla forma, il che è un argomento forte, per cui alcuni hanno concluso che in realtà per Aristotele è la forma il principio di individuazione. Sulla stessa linea di argomentazione, spesso viene citato un altro testo di Aristotele dove lui, rifiutando il concetto pitagorico dellanima secondo il quale essa può entrare in qualsiasi corpo, sottolinea che "è evidente che ogni corpo ha una sua propria forma", ${ }^{13}$ e questo vorrebbe dire che è proprio questa sua forma che lo rende un corpo unico. Inoltre, quando Aristotele in Metafisica si chiede perché una cosa concreta sia l'uomo oppure perché un corpo ha delle caratteristiche specifiche, allora lui sotto questo 'perché cerca "la causa della materia, vale a dire la forma per cui la materia è una determinata cosa: e questa è appunto la sostanza". ${ }^{14}$ E. Regis ritiene che questo testo si riferisce all'individuazione della materia e non della sostanza. Pertanto, per lui questo non sarebbe un argomento che la forma è il principio di individuazione dell'ente. Tuttavia, lesperto massimo della metafisica di Aristotele J. Owens sottolinea che „forma sensibile, se separata, è un 'questo"'. ${ }^{15}$

Però, siccome il principio di individuazione è anche un principio di moltiplicazione, diventa chiaro che la forma non può essere moltiplicata grazie alla materia come tale, ma con una materia quantitativa. Ciò significa che non potranno essere unita qualsiasi materia con qualsiasi forma, o qualsiasi carne con la forma umana per ottenere l'ente umano, ma ci vorrà un certo tipo di carne e di ossa a dare l'uomo. E affinché tale carne dia origine alle persone diverse, esse

12 Cf. Aristotele, De anima, 412 a 8-9. Praticamente la stessa divisione della sostanza che si trova da lui nella Metafisica, 1042 a 26-30.

13 Cf. Aristotel, De anima, 407 b 22-24.

${ }^{14}$ Cf. Aristotele, Metafisica, 1041 b 7-10.

15 J. Owens, The doctrine of being in the Aristotelian Metaphysics, Toronto 1978, ed. Pontifical Institute of Medieval Studies, p. 386. Ciò significa che qua la forma viene considerata come una sostanza. Owens trova la conferma per questa affermazione in libro Metafisica di Aristotele, 1017 b 25 i 1042 a 29. In effetti, Aristotele usa qua la sua famosa espressione tó $\delta$ $\tau \iota$ per indicare la forma, ovvero la determinazione formale. 
„dovranno diversificare nel carattere, vale a dire nella forma. Socrate e Callia, anche se corrispondono nella loro forma specifica, devono differire in forma di loro materia". ${ }^{16}$

Sembra che Aristotele di solito indicava una singola sostanza concreta con

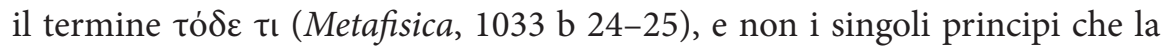
compongono. Tuttavia, in alcuni posti lui usa lo stesso termine ( $\tau$ ó $\delta \varepsilon \tau \iota)$ per indicare la forma, come per esempio quando dice che „in realtà, solamente ciò che è un alcunché di determinato è essenza" (Metafisica, 1030 a 3-5). Perciò E. Regis conclude che Aristotele considerava che sia per la forma sia per l'unione di forma e materia si può dire che sia 'questo qualcosa. ${ }^{17}$ Lo stesso vale per il suo termine synolon. A volte usa questa espressione per indicare la vera sostanza e altre volte la attribuisce alla forma. G. Reale considera che non si tratta di una contraddizione, ma che dipende dal punto di vista. Infatti, dal punto di vista empirico e della costituzione dell'ente il synolon, ovvero un ente singolare e concreto, è la vera sostanza, però dal punto di vista metafisico e speculativo si tratta della forma. ${ }^{18}$ Aristotele eguaglia la forma ed eidos e considera che eidos non diviene e non è universale; diverse volte la indica come tÒde ti „espressione che indica il determinato che si oppone all'universale astratto". ${ }^{19}$

J. Owens respinse la teoria secondo la quale la materia sarebbe il principio di individuazione, perché la materia come tale, siccome indeterminata, non può essere l'origine di determinazione, e quindi non può essere neanche il principio di individuazione dellente. ${ }^{20}$ Lui considera che il problema del principio di individuazione dellente si pone soltanto se la forma viene osservata come universale poiché ciò successivamente richiede un principio che potrebbe ridurla alla individualità. Così lui conclude che secondo Aristotele la forma dellente sensibile in sé non è ne individuale, né universale, neppure rende l'individualità dell'ente complesso e sensibile, piuttosto "lente complesso è individuale, però ciò

${ }^{16}$ D. Ross, Aristotle, London 1995, Routlidge, p. 176. L'autore inoltre ritiene che così si arriva all'essenza di singola persona, la quale in qualche modo oltre la forma specifica include anche caratteristiche stabili come fonte di diversità che formerà individui diversi e che realizzerà la perfezione della specie alla quale appartengono in un modo specifico (cf. ibid. p. 176).

${ }^{17}$ Cf. E. Regis, Aristotle's 'principle of individuation', p. 164. Lui usa la sua espressione "thisses", che potrebbe essere tradotta come "quiddità".

${ }^{18}$ Cf. G. Reale, Storia della filosofia antica, II: Platone e Aristotele, Milano 1997, ed. Vita e Pensiero, p. 431.

19 G. Reale, Storia della filosofia antica, II: Platone e Aristotele, p. 434.

${ }^{20}$ Cf. J. Owens, The doctrine of being in the Aristotelian Metaphysics, p. 332-333. 
sappiamo tramite la sua forma”, tenendo questo in considerazione secondo lui, “ il principio di individuazione " si dimostra un problema molto non-aristotelico". ${ }^{21}$ Anche E. Regis ha raggiunto una conclusione simile nel suo articolo sul problema di individuazione in Aristotele. Secondo lui in Aristotele non cè niente di più primario e concreto della sostanza, composta da forma e materia che non sono niente di più che aspetti distintivi di questa unione. Finalmente, nel suo studio lui conclude che in Aristotele, secondo il quale realmente esistono soltanto le sostanze singole e concrete, il problema di individuazione "non è un problema nel mondo di Aristotele degli enti individuali". ${ }^{22}$

Come si può vedere da quanto detto, risulta che, da un lato la forma determina la materia, ma da secondo lato la forma ha bisogno della materia per essere individuale. Sembra trattarsi di un circolo vizioso. Questo ha animato alcuni studiosi di trovare la soluzione nella unione di forma e materia. È vero che Aristotele considerava che il nostro mondo è il mondo reale e che tutte le sostanze in esso, siano esse sostanze pure separate dalla materia, oppure sostanze sensibili composte di forma e materia, sono in realtà sempre individuali. Tuttavia, il problema sorge proprio qui. Quando osserviamo una sostanza sensibile concreta e individuale, composta di materia e forma, notiamo che la sua forma è identica alla forma di tutte le altre sostanze individuali della stessa specie, per cui non vediamo in che modo sarebbe possibile che la sua forma sia il principio secondo il quale tale specifica sostanza si differenzia dalle altre della stessa specie. Perciò sembrava possibile che sia la materia ad essere il principio di individuazione. Nel caso di sostanze pure e separate si tratterebbe della forma. È proprio questo punto, secondo D. Ross, che ha indotto alcuni filosofi scolastici a dedurre che in tale caso, di tale specie esiste un solo ente. Tuttavia, secondo lui, questo crea alcune difficoltà di interpretazione. ${ }^{23}$

Sembra che D. Ross era riluttante ad accettare la tesi secondo quale la materia sarebbe il principio di individuazione, soprattutto perché è molto strano che sia la materia, inconoscibile in se stessa secondo Aristotele, il principio che determina la specificità di qualsiasi sostanza reale. D. Ross pone la domanda se questo vuole dire allora che tutte le sostanze sensibili non sono

21 J. Owens, The doctrine of being in the Aristotelian Metaphysics, p. 394.

22 Cf. E. Regis, Aristotle's 'principle of individuation', p. 166.

${ }^{23}$ Cf. D. Ross, Aristotle, p. 175-176. Certamente D. Ross si riferisce qui principalmente a Tommaso d'Aquino. 
conoscibili fino in fondo. ${ }^{24}$ Perciò egli suggerisce la sua interpretazione, ovvero che il principio di individuazione secondo Aristotele era una materia concreta e forma insieme. Il testo di Aristotele sul quale basa la sua tesi è il seguente: „e anche delle cose che rientrino sotto la stessa specie le cause saranno diverse, non specificamente diverse, ma numericamente diverse nei diversi individui: la tua materia, la tua forma e la tua causa efficiente numericamente non sono identiche alle mie, mentre sono universalmente e specificamente identiche". ${ }^{25}$ Bisogna notare però che $\mathrm{E}$. Regis usa questo stesso testo come argomento per la tesi che il principio di individuazione è la forma, e quest è per noi un segno chiaro che sarà necessario cercare un principio più profondo, che in qualche modo individuerà sia la materia che la forma, e attraverso di loro anche un ente completo e complesso.

Il pensiero metafisico medievale ha continuato ad esaminare questo problema con l'intenzione di scoprire il principio di individuazione di un individuo particolare. ${ }^{26}$ Durante questo periodo sono emerse varie teorie e proposte per una soluzione, ma noi ci limiteremo ad esaminare soltanto le più importanti.

\section{Gli accidenti come principio di individuazione}

Per molti studiosi medievali era illogico che la materia, che appartiene a tutti gli enti sensibili e non presuppone le distinzioni in se stessa, sia un principio così importante, che individua ogni ente per se stesso in rapporto con la specie. ${ }^{27}$

In effetti, ciò che ci fa distinguere Aristotele da Platone sono esattamente gli accidenti di Aristotele: quando e dove ha vissuto, chi erano i suoi genitori, cosa e come ha insegnato, ciò che ha scritto e così via. Tuttavia, dobbiamo subito

${ }^{24}$ Cf. D. Ross, Aristotle, p. 176. Aristotele nella sua Metafisica dice espressamente che „la materia è di per sé inconoscibile" (Metafisica, 1036 e 8).

${ }^{25}$ Aristotele, Metafizica, 1071 a 27-29. Questo è meglio capito nel caso dell'uomo. È vero che il corpo di Pietro è identico al corpo di Paolo per specie, infatti stiamo parlando del corpo umano, ma la mano di Pietro è una cosa, e la mano di Paolo un'altra cosa. È vero che i genitori di Pietro sono uomini, come lo sono quelli di Paolo, ma sono uomini diversi, ovvero persone diverse.

${ }^{26}$ Cf. P. King, The Problem of Individuation in the Middle Ages, http://individual.utoronto. ca/pking/articles/Mediaeval_Individuation.pdf (15.01.2014).

${ }^{27}$ Cf. R. Meßner, Il principio di individuazione nella visione scotista, „Idee” 64 (2007), p. 21. 
dire che gli accidenti qui sono in funzione epistemologica, e che non ci danno risposte sul principio metafisico. Questa soluzione indica come un argomento il fatto che Aristotele e Platone possiedono pienamente la stessa essenza, cioè lessenza umana, e se differiscono, allora gli elementi di differenziazione non devono essere ridotti alla loro essenza, e quindi si tratta alloro dei loro accidenti. Il filosofo più importante che ha sostenuto questa teoria era S. Boezio. Egli ritiene che sono gli accidenti a determinare che un ente diventi una cosa concreta che occupa uno spazio concreto, il quale è uno degli accidenti di questa cosa concreta. Ricordiamo il suo famoso testo: „Ora, la varietà degli accidenti provoca diversità quanto al numero: infatti tre uomini non sono diversi né per il genere né per la specie ma per i loro accidenti: poiché, anche se separiamo da essi col pensiero tutti gli accidenti, tuttavia per ognuno di loro il luogo è diverso, e non possiamo in alcun modo ridurlo ad uno solo: dal momento che due corpi non potranno occupare uno stesso luogo, e il luogo è un accidente. Poiché dunque son resi molti dai loro accidenti, sono anche molti quanto al numero". 28

Molti hanno accettato questa teoria, come per esempio Giovanni Scoto Eriugena e Anselmo d'Aosta. Sembra che è stata la teoria dominante per secoli, oppure come l'ha definito J. Gracia "teoria standard di individuazione". ${ }^{29} \mathrm{Va}$ sottolineato che, dopo Boezio, vengono sviluppare due, cosiddette "tradizioni" filosofiche per quanto riguarda il problema di individuazione. Una è orientata più verso l'aspetto metafisico di questo problema. I suoi rappresentanti principali sono stati G. Scoto Eriugena, Oddone di Tournai, Teodorico di Chartres e Gilbert di Poitiers. Jorge J. E. Gracia sottolinea il valore di Gilbert di Poitiers quando dice di lui che „senza dubbio, nessuno altro aveva la visione metafisica di individualità più sofisticata, tra quelle che ci sono rimaste dal Medioevo precoce. E il suo punto di vista non è soltanto sottile, ma anche originale, dal momento che radicalmente devia dalla teoria standard di individualità,

${ }^{28}$ Cf. Boethius, De Trinitate, I: “Sed numero differentiam accidentium uarietas facit. Nam tres homines neque genere neque specie sed suis accidentibus distant; nam uel si animo cuncta ab his accidentia separemus, tamen locus cunctis diuersus est quem unum fingere nullo modo possumus; duo enim corpora unum locum non obtinebunt, qui est accidens. Atque ideo sunt numero plures, quoniam accidentibus plures fiunt"; tal. pr.: S. Boezio, La consolazione della filosofia. Gli opuscoli tologici, Milano 1979, ed. Rusconi, p. 360-361.

${ }^{29}$ Cf. J. J. E. Gracia (a cura di), Individuation in Scholasticism: The Later Middle Ages and the Counter-Reformation (1150-1650), Albany 1994, ed. State University of New York, p. 543. 
accettato da quasi tutti del suo tempo" ${ }^{30}$ Tuttavia, dopo ci saranno dei studiosi che criticheranno questa teoria.

Va notato che lo stesso Boezio cercava un'ulteriore elemento per avvalorare il ruolo di accidenti nell'individuazione dell'ente. Così, nella citazione di sopra lui espone le loro „diversità", come elemento aggiuntivo, da un lato, e dall'altro ci offre la possibilità di ridurre la funzione di individuazione ad un unico accidente - lo spazio. P. King ha riconosciuto questo come una locazione spazio-temporale. ${ }^{31}$

Nel XII secolo, Pietro Abelardo ha criticato severamente questa teoria, e dopo questo pochi la condividevano. L'essenza della sua critica è che questa teoria de facto agli accidenti attribuisce una priorità ontologica rispetto alla sostanza e questo è sbagliato. Infatti, nella essenza degli accidenti sta che loro sono ulteriori determinazioni di una sostanza, ovvero di un soggetto. ${ }^{32}$ Effettivamente, è inaccettabile che le singole caratteristiche di un individuo determinano la sua individualità, ma allo stesso tempo non sono la parte costituente di quell'individuo. Questa tesi in seguito sarà respinta anche da G. Duns Scoto con l'affermazione che gli incidenti da soli non possono produrre una distinzione numerica. $^{33}$

${ }^{30}$ J. J. E. Gracia, Introduction to the problem of individuation in the early Middle Ages, München - Wien 1984, ed. Philosophia Verlag, p. 175-176.

${ }^{31}$ Cf. P. King, The Problem of Individuation in the Middle Ages, http://individual.utoronto. ca/pking/articles/Mediaeval_Individuation.pdf (07.12.2013).

${ }^{32}$ Pietro Abelardo riporta questa critica nella sua opera Logica (cf. Logica ingredientibus: 1.01.: „Illud quoque stare non potest quod indiuidua per ipsorum accidentia effici uolunt. Si enim ex accidentibus indiuidua esse suum contrahunt, profecto priora sunt eis naturaliter accidentia sicut et differentiae speciebus quas ad esse conducunt").

${ }^{33}$ G. Duns Scoto, Il principio di individuazione. Ordinatio II, d. 3, pars 1, q. 4,: „sed no faciunt primam differentiam (sed alia est, prior, differentia numeralis), neque ipsa sola faciunt differentiam numeralem"; tal. pr.: G. Duns Scoto, Il principio di individuazione. Ordinatio II, d. 3, pars 1, Quaestiones 1-7, Napoli 2011, ed. Il Mulino, p. 65. Duns Scoto spiega ulteriormente che è necessario tenere a mente l'intenzione di Boezio, che secondo lui è stata quella di dimostrare che non ci sono differenze numeriche nelle persone divine. Perciò Boezio ha sostenuto che gli accidenti producono la differenza numerica, ma siccome nelle persone divine non ci sono gli accidenti, allora non cè neanche differenza numerica. Tuttavia, Duns Scoto considera che la differenza tra gli accidenti non causa la differenza numerica, ma segue questa differenza condizionata da un altro principio (ibid.: „Dico quod omnem distinzione numerale concomitatur distinctie accidentuim"). Ne consegue quindi che la differenza tra gli accidenti in qualche modo segue anche la differenza numerica. 


\section{Forma come principio di individuazione}

Seguendo la idea sopracitata, ovvero che il principio che porterebbe l'individualità ad un ente dovrebbe essere costitutivo per tale essere, alcuni studiosi hanno pensato di semplicemente aggiungere che tale principio dovrebbe essere formale. Quindi, secondo loro il principio di individuazione di un ente è la forma sostanziale. Naturalmente stiamo parlando di una forma individuale, non di una forma universale. E quindi, così come la razionalità entra nella specie di animali per costruire una specie razionale, un animale razionale, così la forma individuale entra nella specie particolare per individuare un ente concreto e specifico di quella specie. Il genere, quindi, viene ridotto alla specie tramite differenze formali, e una specie è ridotta all'individuo tramite le differenze di individualizzazione. ${ }^{34}$

Il vantaggio di questa interpretazione sta nel fatto che il principio ricercato è connesso con l'essenza di un ente in un modo più diretto, se la forma è quella che da l'essenza. Un vantaggio particolare si vede quando si tratta l'uomo considerato come una persona e ciò realmente può essere considerato come una novità del pensiero filosofico medievale. Infatti, questa teoria rende più facile identificare l'anima personale delluomo come la forma del suo corpo. I rappresentanti più importanti di questa teoria sono Averroè, Godefridus de Fontibus e Jedi Ben Abraham Bedersi. Alcuni annoverano anche Boezio tra i rappresentanti di questa teoria, anche se nelle sue opere non si trova il testo esplicito che giustifichi tali affermazioni.

Lobiezione principale aggiudicata a questa teoria è che, nonostante renda Aristotele e Platone individui diversi, ma non secondo la loro essenza. Se la loro forma li individuasse secondo l'essenza, allora ognuno di loro sarebbe una specie particolare, similmente come è il caso degli angeli secondo Tommaso d’Aquino, ma che per Aristotele e Platone chiaramente questo non vale.

\section{Materia come principio di individuazione}

Generalmente si ritiene che il principale rappresentante medievale della teoria che il principio di individuazione è la materia fosse stato Tommaso d'Aquino.

${ }^{34}$ Cf. P. King, The Problem of Individuation in the Middle Ages, http://individual.utoronto. ca/pking/articles/Mediaeval_Individuation.pdf (07.12.2013). 
Pertanto valuteremo criticamente la sua opinione per vedere se ci sono delle idee originali che potrebbero dare un contributo significativo alla soluzione di questo grande ed importante problema, soprattutto per quanto riguarda la persona umana.

Innanzitutto bisogna capire cosa san Tommaso considerava un individuo. È nota la sua definizione che dice „l'individuo poi è ciò che è indistinto in se stesso e distinto dagli altri”. ${ }^{35}$ È evidente che in questo mondo cè una moltitudine di enti e che ogni cosa è diversa dall'altra. Questo si esprime quando si dice che ogni cosa è qualcosa (aliquid). La parola latina aliquid significa in primo luogo aliud quid, ovvero qualcos'altro. Infatti, per quanto riguarda un ente, così come viene detto di essere 'uno' (unum), in quanto è indivisibile in se stesso, così allo stesso modo viene detto di essere 'qualcosa d'altro' (aliquid) in quanto diverso dagli altri enti. ${ }^{36}$ Così come 'uno' è la parte trascendentale degli enti, così per san Tommaso sarà anche questo' qualcos'altro' (aliquid). Il concetto stesso di diversità si trova già in antichità, in Platone, Aristotele, Plotino, Proclo e, infine, in Porfirio. Tuttavia, come abbiamo già dimostrato, Tommaso d'Aquino annovera la diversità tra gli trascendentali dellente, il che significa che appartiene ad ogni ente, cioè, ogni ente è un'altra cosa rispetto a tutti gli altri enti. Questo trascendentale ha sempre attirato l'attenzione degli studiosi del pensiero di san Tommaso, ma non un'uniformità di interpretazione. Ma nonostante le diverse interpretazioni, da quella che dice che aliquid indica una individualità radicale dell'ente (R. Schmitz), a quella che dice che indica la moltiplicazione interna dellente (G.Ventimiglia), B. Mondin ritiene che queste interpretazioni sono compatibili e che indicano un unico principio dellente, e cioè l'essere (esse) in essi. ${ }^{37}$

Nel suo pensiero su questo problema, Tommaso d'Aquino inizia dalla concezione aristotelica della materia come principio di individuazione. Infatti, in molti luoghi lui conferma questa teoria, come ad esempio quando dice che ogni cosa viene individuata in base alla materia (ex materia). ${ }^{38}$ In un altro punto era molto più esplicito, dicendo che „la materia è infatti il principio di

${ }^{35}$ Tommaso d'Aquino, S. Th., I, q. 29, a. 4, c: „Individuum autem est quod est in se indistinctum, ab aliis vero distinctum".

36 Cf. Tomasso d'Aquino, QD De Ver., q. 1, c.

${ }^{37}$ Cf. B. Mondin, La metafisica di S. Tommaso d'Aquino e i suoi interpreti, Bologna 2002, Edizioni Studio Domenicano, p. 463-466.

${ }^{38}$ Cf. Toma Akvinski, De ente et essentia, c. 6. 
individuazione". ${ }^{39}$ Oppure quando compara il suo ruolo con il ruolo della forma dicendo „poiché il principio di individuazione è appunto la materia, come la forma è principio della specie". ${ }^{40}$ Abbiamo detto che lui parte da Aristotele, però riporta anche un contributo proprio alla soluzione di questo problema, portando una precisazione molto importante che non si tratta di una materia qualsiasi, oppure di materia considerata in modo astratto, ma della materia caratterizzata dalla quantità (materia signata quantitate), ovvero la materia determinata dalle dimensioni concrete o dall'estensione.

Si potrebbe dire che lui ha fatto una sintesi di quello che la tradizione filosofica prima di lui ha già riflettuto, e che l'ha complementato con chiarezza che caratterizza il suo pensiero filosofico. Infatti, varie volte lui dice esplicitamente „il principio di individuazione è la materia quantitate signata”. ${ }^{41}$ Ciò significa la materia caratterizzata dalla quantità, cioè, non materia prima, ma la materia che è già dotata di una forma, che realizza la sua quantità.

La quantità è una delle proprietà della sostanza materiale per la quale esse occupano un certo spazio, cioè possiedono l'estensione e dimensione. Per la stessa singolarità, esse possiedono le parti al di fuori dai parti (partes extra partes) e per questo sono divisibili. Pertanto, si dice che un ente materiale sia ens quantum, cioè un ente quantificato, perché possiede una estesa, ovvero misurabile, quantità. ${ }^{42}$ Vogliamo dire subito che qualsiasi ente materiale, dotato di quantità estesa, già per questo motivo è divisibile, misurabile e individuale. La prima radice della moltiplicabilità degli enti materiali è nella quantità estesa, poiché se venisse meno la quantità dell'ente, esso sarebbe indivisibile. ${ }^{43}$ Pertanto M. Artigas e J. J. Sanguineti concludono risolutamente che „la radice ultima dell'individualità negli enti materiali è la quantità estesa”, e che, „la materia quantitate signata è il principio individuante le forme sostanziali corporee". ${ }^{44}$

39 Tommaso d'Aquino, Super Boetium De Trinitate, q. 5, a. 2.: „materia enim est individuationis principium".

40 Tommaso d'Aquino, C.G., IV, c. 63.

41 Tommaso d'Aquino, S. Th., I, q. 75, a. 4; III, q. 77, a. 2; C. G., IV, c. 65.

${ }^{42}$ Cf. M. Artigas - J. J. Sanguineti, Filosofia della natura, Firenze 1989, Le Monnier, p. $126-$ 127. Diciamo che esiste anche una "quantità numerica” che separa l'ente dall'ente e che ci permette la numerabilità degli enti. L'idea che l'ente materiale sia divisibile a causa della sua quantità ci viene da Aristotele (cf. Metafisica, 1020 a 7).

${ }^{43}$ Cf. Tommaso d'Aquino, C.G., IV, c. 65; Aristotele, Fisica, 186 b 12-33.

${ }^{44}$ M. Artigas - J. J. Sanguineti, Filosofia della natura, p. 138. 
Il famoso tomista italiano, B. Mondin, ha notato che san Tommaso „perfeziona la tesi aristotelica relativa al principio di individuazione, precisando che non è la materia qua talis a svolgere il ruolo della individuazione delle forme, ma la materia quantitate signata". ${ }^{45}$ San Tommaso ha perfezionato la tesi di Aristotele anche in vista del ruolo limitante della materia in relazione alla forma. Per lui, la materia non è un potenziale principio di tutte le forme, ma soltanto delle forme di sostanza sensibili, e di conseguenza neanche la materia quantitate signata non può essere il principio di individuazione degli enti che non possiedono la materia. Con questo l'insegnamento, san Tommaso si allontanava molto dai suoi contemporanei, che sulle tracce di Avicebron hanno insegnato ilemorfismo universale.

Il suo insegnamento sulla materia caratterizzata dallestensione san Tommaso dimostra di essere consapevole del problema che abbiamo notato quando abbiamo riflettuto il pensiero di Aristotele, ovvero che la materia individua la forma, ma che anche la forma in qualche modo individua la materia. Egli dice che Aristotele considera che alcune cose sono uno per il numero, altro per la specie e altro ancora per il genere, e alcuni per analogia. „Sono uno per il numero quelle la cui materia è una sola: infatti la materia, in quanto sotto le dimensioni che la contrassegnano, è il principio dell'individuazione della forma; per questo, il singolare ha dalla materia il fato di essere uno per il numero diviso dagli altri”. ${ }^{46}$

Il suo insegnamento chiaramente esprime che la materia fornisce allente un individualità numerica, poiché "tutte le cose che sono identiche nella specie e diverse nel numero implicano la materia”, e poi continua, „infatti la diversità che proviene dalla forma implica diversità di specie; quella invece che proviene dalla materia implica diversità numerica". ${ }^{47} \mathrm{Nel}$ suo commento del De Trinitate di Boezio, lui dettagliatamente riporta come fa la materia ad individuare la forma, quando dice che "come le parti del genere e della specie sono materia e forma, così le parti dell'individuo sono questa materia e questa forma, per cui come le diversità nel genere e nella specie è prodotta dalla diversità della materia o della forma, considerate in senso assoluto, così la diversità numerica è prodotta da questa forma e da questa materia ... Ed è in virtù di tali dimensioni

${ }^{45}$ B. Mondin, La metafisica di S. Tommaso d'Aquino e i suoi interpreti, p. 289. Quindi si tratta di una materia che è stata già quantificata, ovvero marcata con l'estensibilità.

46 Tommaso d'Aquino, In Metaph., lib. 5, lec. 8, br. 876.

47 Tommaso d'Aquino, C.G., II, c. 93. 
indeterminate che la materia viene resa questa materia segnata, e può a sua volta rendere individuale la forma. Questo è dunque il modo in cui la materia produce la diversità numerica in una stessa specie", e poi aggiunge un'osservazione importante per quanto riguarda la differenza tra 'materia' e 'questa materia', „appare perciò che la materia presa in se stessa non è principio di diversità né secondo la specie, né secondo il numero".48

Quando osserviamo la forma degli enti corporei per se stessa, in generale, essa non ha bisogno della materia per esistere, siccome essa stessa è la portatrice dell'essere, ma „ha bisogno della materia quando è una forma tale che senza di essa non può sussistere". ${ }^{49}$ Pertanto, è necessario dire che „le forme sono individuate dalla propria materia." ${ }^{50}$ Infatti, nel suo commento di De anima di Aristotele, Tommaso afferma chiaramente che „l'individuazione dell'essenza comune dipende negli esseri corporei e materiali dalla materia corporea racchiusa entro determinate dimensioni". ${ }^{51}$ Altrove lui spiega perché la forma non può essere individuata con la materia in generale, ma deve essere 'questa' o 'quella' materia, ovvero la materia che viene determinato dalla quantità, perché la materia è divisibile solo per la sua quantità. ${ }^{52}$

Tuttavia, per avere una materia che sia caratterizzata dalla estensione e che sia in grado di individuare la forma è necessario che essa stessa sia in qualche modo già individualizzata. Infatti la materia caratterizzata dalla estensione è la materia che in qualche modo è già individualizzata dalla forma che ha dato ad un ente sensibile. Ciò significa che affinché la carne e le ossa, presi in generale, diventino il corpo e le ossa di Pietro, deve realizzarlo la forma di Pietro, ovvero la sua anima. Ma, d’altra parte, affinché la forma umana diventi l’anima di Pietro, ci vogliono anche il corpo e le ossa di Pietro che lo realizzano, perché „la

48 Tommaso d'Aquino, Super Boetium De Trinitate, q. 4, a. 2, c.

49 Tommaso d'Aquino, QD De Anima, q. 6, c.: „sed indiget materia cum sit tolis forma quae per se non subsistat".

50 Tommaso d'Aquino, QD De Anima, q. 1, arg. 2.

51 Tommaso d'Aquino, In De Anima, lib. II, lect. 12: „Individuatio autem naturae communis in rebus corporalibus et materialibus, est ex materia corporali, sub determinatis dimensionibus contenta". Qua con il circoscrizione dimensionale si intende alla circoscrizione spazio-tempo, e la frase ti Tommaso materia sub determinatis dimensionibus contenta è il sinonimo per materia quantitate signata.

${ }^{52}$ Cf. Tommaso d'Aquino, Super Boetium De Trinitate, pars 2, q. 4, a. 2, co. 6: „Non enim forma individuatur per hoc quod recipitur in materia, nisi quatenus recipitur in hac materia distincta et determinata ad hic et nunc". 
materia infatti è il principio di individuazione delle forme". ${ }^{53}$ In un commento della Metafisica di Aristotele, egli scrive che l'uomo, considerato generalmente, è composto di corpo e anima, ma non di 'questo corpo' e 'quest'anima'; mentre invece qualcosa di individuale indica qualcosa complesso composto „'dall'ultima materia' (ex ultima materia), cioè dalla materia individuale, in quanto Socrate è qualcosa composto da quest'anima e da questo corpo (hoc anima et hoc corpore). Lo stesso accade negli altri singolari”. ${ }^{54}$ Pertanto, la radice della singolarità di questi enti particolari sarà una materia individuale, e non una materia considerata generalmente. ${ }^{55}$

È stato già detto che la materia signata quantitate è il principio di individuazione, ma è anche il principio di moltiplicazione. Per questo gli enti della stessa specie si moltiplicano in realtà attraverso l'individuazione e la capacità della moltiplicazione è basata sulla quantità, ovvero sulla divisibilità della materia. ${ }^{56}$ La natura fisica, in contrapposizione a quella spirituale, è moltiplicabile, perché la loro attualità è incompleta, il che significa che la loro essenza non risiede soltanto nella loro forma, ma comprende anche la materia. Perciò, per gli enti spirituali si dice che sono di forma pura, o come Aristotele li chiamava, sostanze separate. Vale a dire, separate dalla materia, ovvero non hanno materia. Secondo Tommaso, loro sono „sussistenti per se stesse, sono anche individuali per se stesse". ${ }^{57}$ Ovviamente si tratta ancora una volta della materia che possiede estensione e non della materia in generale, poiché „soltanto la quantità, o estensione implica l'elemento da cui può derivare la pluralità degli individui nella medesima specie”, che vale a dire che, „la prima radice di codesta molteplicità sembra derivare dallestensione". ${ }^{58}$ Egli ha riflettuto anche sullo scopo di questa moltiplicazione degli enti, ma non è andato oltre quanto detto da Platone e Aristotele. Per cui, anche secondo lui il significato di questa moltiplicazione sta nel fatto che la natura di una specie non può essere

53 Tommaso d'Aquino, S. Th., I, q. 75, a. 5, c.

${ }^{54}$ Tommaso d'Aquino, In 12 libros Metaphysicorum Aristotelis expositio, lib. 7, lec. 10, n. 1490

${ }^{55}$ Cf. Tommaso d'Aquino, S. Th., I, q. 86, a. 1, c: „quia principium singularitatis in rebus materialibus est materia individualis."

${ }^{56}$ Cf. Tommaso d'Aquino, C.G., IV., c. 65.

57 Tommaso d'Aquino, S. Th., I, q. 77, a. 2, c.

${ }^{58}$ Tommaso d'Aquino, C.G., IV, c. 65.: „Et quia sola quantitas dimensiva de sui ratione habet unde multiplicatio individuorum in eadem specie possit accidere, prima radix huiusmodi multiplicationis ex dimensione esse videtur." 
preservata in un unico ente concreto, ma piuttosto in una varietà degli individui che formano questa specie. ${ }^{59}$

Lobiezione principale di questa teoria deriva dalla nostra esperienza sensibile quotidiana che ci da l'evidenza che ogni ente sensibile intorno a noi ha delle dimensioni e quindi occupa un certo spazio. In effetti, possedere la dimensione è un fatto comune e ovvio per tutti gli enti sensibili. Pertanto, sembra infondato che l'individualità di un individuo viene spiegata attraverso un fatto così ovvio e un elemento comune di tutti gli enti sensibili, che sembra essere una conseguenza, piuttosto che il principio di individuazione. ${ }^{60}$

Consideriamo che questa obiezione non sia vera, perché san Tommaso era ben consapevole del fatto che sola materia non può pienamente determinare l'individualità di un ente, e soprattutto non la persona umana. È vero che lui non ha indagato ulteriormente questo problema, ma riteniamo che ci siano abbastanza elementi nel suo pensiero metafisico per continuare la ricerca della soluzione al problema di individuazione in questa direzione. Riteniamo che per ricerche ulteriori è utile iniziare dal punto in cui lui spiega in che senso il termine persona può essere attribuito all'uomo che è uno, e a Dio che è uno, sebbene ha tre persone. Innanzitutto, egli distingue il termine 'persona', il che indica il termine generico, da 'quella persona', il che indica un individuo specifico, ad esempio una persona divina. Dopo aver esposto le definizione di persona di Boezio: sostanza individuale di natura razionale, egli definisce l'individuo come "ciò che è indistinto in se stesso e distinto dagli altri" é1 e quindi conclude che la persone di qualsiasi natura, umana, angelica o divina, indica ciò che è diverso (quod est distinctum) in quella natura. E nel caso di un uomo singolare, per esempio Pietro, questo vuol dire "questa carne, queste ossa, quest'anima, che sono il principio di individuazione dell'uomo" ${ }^{62} \mathrm{E}$ anche se queste cose: 'questo corpo', 'queste ossa' e 'questanima', non fanno parte del significato del termine persona, esse fanno parte del significato di 'persona umana' È proprio questa transizione a persona come elemento di differenziazione di individui umani, gli uni dagli altri, che ci suggerisce la conclusione che anche san Tommaso era

59 Cf. Tommaso d'Aquino, C.G., II, c. 93. Questo spiega anche la capacità dell'ente vivente di moltiplicarsi e anche la brama per la conservazione della specie.

${ }^{60}$ Cf. P. King, The Problem of Individuation in the Middle Ages, http://individual.utoronto. $\mathrm{ca} /$ pking/articles/Mediaeval_Individuation.pdf (07.12.2013).

61 Tommaso d'Aquino, S. Th., I, q. 29, a. 4, c.

${ }^{62}$ Tommaso d'Aquino, S. Th., I, q. 29, a. 4, c.: „has carnes et haec ossa et hanc animam, quae sun principia individuantia hominem." 
consapevole della necessità di trovare un nuovo principio di individuazione, che sarebbe metafisicamente più profondo della materia e della forma. San Tommaso teneva sempre in mente la necessità che la materia e la forma devono essere già individuati, che devono essere 'questo qualcosa'. Così, nel suo commento della De Trinitate di Boezio egli scrive che così come la materia e la forma sono le parti del genere e della specie, „così le parti dell'individuo sono questa materia e questa forma". ${ }^{63}$

Che il principio che cerchiamo potrebbe essere actus essendi di Tommaso ce lo indica un testo notevole della sua opera QD De anima, dove lui spiega il rapporto fra l'individualità dell'ente e il suo atto di essere, e dice chiaramente che „la ragione dell'essere e dell'individuazione di ogni cosa è la medesima”. ${ }^{64}$ Proprio qui dovremmo cercare un collegamento dell'insegnamento che actus essendi è il principio di individuazione di una persona singola e completa con lidea di Duns Scoto di un unico principio di individuazione. Se si tratta di haecceitas o no rimane una questione da indagare. Proprio la nostra tesi che sia actus essendi il principio finale di individuazione spiega che la sola materia non può essere quella che individua la persona. Pertanto, con il deterioramento del corpo non si ferma l'individuazione dell'anima umana e l'anima di una concreta persona umana, per esempio Pietro e Paolo, non viene disintegrata in qualche forma generale dell'anima dopo la morte biologica del corpo, ma continua ad essere come l'anima individuale di Pietro o Paolo. ${ }^{65}$

Tuttavia, da ciò non si dovrebbe trarre la conclusione che ogni ente è un individuo per se stesso, nel senso che non ha nulla in comune con gli altri enti. Anzi, tutti gli enti sensibili hanno un principio comune che li colloca nella stessa specie e perciò essi devono avere un altro principio che li differenzia all'interno della specie, che li rende individui tra loro e che è incomunicabile a qualsiasi altro ente. ${ }^{66}$ P.-B. Grenet ritiene che, nel caso di Dio e degli enti spirituali puri, „diviene dunque evidente che il principio dell'individuazione è il principio della

${ }^{63}$ Tommaso d'Aquino, Super Boetium De Trinitate, q. 4, a. 2.

${ }^{64}$ Tommaso d'Aquino, QD De Anima, q. 1, ad. 2.: "Ad secundum dicendum quod unumquodque secundum idem habet esse et individuationem."

${ }^{65}$ Cf. Tommaso d'Aquino, QD De Anima, q. 1, ad. 2.: „Sicut igitur esse animae est a Deo sicut a principio activo, et in corpore sicut in materia, nec tamen esse animae perit pereunte corpore; ita et individuatio animae, etsi aliquam relationem habeat ad corpus, non tamen perit corpore pereunte."

${ }^{66}$ Cf. Tommaso d'Aquino, S. Th., I, q. 11, a. 3, c.: „non potest communicari nisi uni tantum”. E l'atto di essere proprio è effettivamente intercomunicabile ad un altro ente. 
determinazione dell'essere: in una parola, 'la forma' ${ }^{67}$ Bisogna avvertire che sarebbe sbagliato identificare completamente il principio di individuazione con il principio dellessere negli enti creati, perché solamente in Dio coincidono pienamente il principio di individuazione con il principio di essere. ${ }^{68} \mathrm{Nel}$ caso degli angeli, il principio di individuazione coincide con il principio di specificazione, cioè con la quiddità (quidditas subsistens). Qui dobbiamo stare attenti, perché spesso accade che, nel caso di Dio e in generale nel caso delle forme sussistenti, il principio di individuazione viene confuso con il principio di specificazione, che si identifica con l'intero ente. Pertanto, il principio di individuazione deve essere un principio che fa parte di quellente particolare, ma allo stesso tempo costituisce un ostacolo affinché la perfezione della specie viene attualizzata in quell'individuo.

\section{Materia e forma come principio di individuazione}

Queste domande e dilemmi hanno risultato con il fatto che alcuni hanno sostenuto la teoria della materia e della forma come il principio di individuazione. Il rappresentante principale di questa teoria era san Bonaventura. Egli riteneva che la materia e la forma insieme formano un'unità sostanziale, un ente concreto, e quindi che individuano tale ente in relazione con il genere e con la specie. Se ci chiedessimo perché un ente è un individuo, san Bonaventura risponderebbe che è così perché quest'ente è 'questo-qualcosa'. In questa determinazione di 'questo-qualcosa', la materia avrebbe un ruolo maggiore nel determinare che tale ente sia 'questo', perché essa ha fatto che la forma concreta di tale ente sia localizzata nello spazio e nel tempo. Dall'altro lato, la forma avrebbe un ruolo maggiore che tale ente concreto sia 'qualcosa'. Pertanto, lui conclude che "nel caso delle creature, l'individuazione deriva da due principi", 69 cioè dalla materia e dalla forma.

San Bonaventura riteneva che, a causa della sua mutabilità e passività, che derivano dalla materia, una sostanza è hoc aliquid. E grazie alla forma e alla

67 P.-B. Grenet, Ontologia, Brescia 1967, ed. Paideia, p. 111.

${ }^{68}$ Cf. Tommaso d'Aquino, S. Th., I, q. 11, a. 3, c.: „Secudum igitur idem est Deus, et hic Deus."

69 Bonaventura, In II Sent., d. 3, pars 1, art. 2, q. 3, resp.: „Individuatio igitur in creaturis consurgit ex duplici principio." 
materia, essa è una singola cosa, concreta e sussistente. É. Gilson, un grande conoscitore del pensiero di san Bonaventura, ha concluso che san Bonaventura, per quanto riguarda il problema del principio di individuazione, ha ritenuto che „'individuazione non è possibile senza materia, e tuttavia non è la materia, ma l'unione della forma e della materia a costituire l'individuo come tale" ${ }^{70}$

Tuttavia, sembra che pur impostando due principi di individuazione degli enti, san Bonaventura non ha risolto il problema significativamente. Infatti, rimane incerto come la materia può posizione la forma nello spazio e nel tempo, se questa non è già una forma individuale, e dall'altro canto come la forma può attualizzare la materia, se questa non è già individuato tramite le sue dimensioni. Questo diventa ancora più evidente se prendiamo in considerazione come san Bonaventura comprendeva la materia, che di per sé non è né materiale né spirituale, ma dipende dalla forma che prende. ${ }^{71}$ Ciò significa che la materia non è materia senza la forma. Infatti, san Bonaventura pensava che Dio, fin dall'inizio, ha creato la materia con una forma iniziale ${ }^{72}$ che sarebbe qualcosa come una costante aspirazione verso una forma definitiva, con la quale questa materia formerà un ente individuale concreto. Ciò significa che la forma finale dellente non si unisce con la materia prima, per formare un ente concreto, piuttosto essa perfeziona una certa materia che possiede una forma inferiore. Va notato che per san Bonaventura il ruolo della materia non è soltanto di ricevere qualche forma, ma essa già dentro di sé possiede il germe di una forma futura, che essa può attualizzare, ovvero ciò vuol dire che "nella materia esiste qualcosa di concreato da dove, la causa naturale, quando in essa agisce, mette in evidenza la forma". ${ }^{73}$

\section{Haecceitas come principio di individuazione}

G. Duns Scoto ha dedicato una grande attenzione al problema di individuazione, in particolare al fine di preservare il valore e la dignità della persona umana. Era consapevole che il principio di individuazione si riferisce al fatto che, da

70 É. Gilson, La filosofia di San Bonaventura, Milano 1995, ed. Jaca Book, p. 297.

${ }^{71}$ Bonaventura, In II Sent., d. 3, pars 1, art. 1, q. 2.: „la materia, osservata in sé, non è né spirituale, né materiale."

72 Cf. M. Fumagalli Beonio Brocchieri - M. Parodi, Povijest srednjovjekovne filozofije, Kršćanska sadašnjost, Zagreb 2013, p. 281.

${ }^{73}$ Cf. M. Fumagalli Beonio Brocchieri - M. Parodi, Povijest srednjovjekovne filozofije, p. 282. 
un lato, più individui della stessa specie differiscono uno dall'altro, e dall'altra parte, che vi sia una reale possibilità che una stessa essenza possa essere in diversi enti singolari. Lui prendeva in considerazione l'individuo in modo molto più positivo, come la possibilità che la perfezione della specie si attualizzi nella pienezza. Ciò significa che un nuovo individuo non è solo una ripetizione del tipico rappresentativo della specie, ma porta dentro se una nuova ricchezza che si può attualizzare. La sua creazione arricchisce la realtà, e questo diventa particolarmente evidente nel caso di una nuova persona umana.

Siccome nella tradizione prima di lui la materia aveva più probabilità di essere il principio di individuazione, e infatti anche Tommaso d'Aquino in qualche modo era su questa strada, Duns Scoto ha innanzitutto esaminato la posizione della materia nei confronti di questo problema. In primo luogo, si chiede se la materia fosse individuale per la sua natura. A prima vista, la risposta sembra molto semplice, anche con certa dose di meraviglia perché chiederselo quando è evidente che incontriamo la materia sempre coma una cosa singolare, e quindi non ha bisogno di un ulteriore elemento per individuarla. Poiché la materia è reale è sempre anche singolare, mentre, al contrario, una realtà universale, a prescindere dal livello della realtà che ha, rimane sempre e soltanto una realtà compresa nella mente. Però la questione non è così semplice come sembra, perché Duns Scoto ritiene che tra la reale unità di enti singolari, cioè la unità numerica, e pura universalità esiste un'unità di enti inferiore all'unità numerica. ${ }^{74}$ Quale sarebbe questa unità ed a quali enti si riferisce? Riflettendo sul rapporto tra il genere, la specie e i singoli enti reali, con l'intenzione di comprendere il pensiero autentico di Aristotele, lui arriva alla conclusione che non si può confrontare unità concettuale del genere che si riferisce alle specie con il reale unità della specie comune a tutti gli individui di quella specie. Questo è quello che lui chiama la natura comune (natura communis). ${ }^{75}$ E questa unità della natura, vista per se stessa, non determina l'unità dei singoli enti concreti.

Questa natura precede tutti i possibili modi in cui essa può esistere, essa è semplice essenza, quod quid est, non è né universale, né individuale. Perciò Duns Scoto respinge la tesi secondo la quale la sostanza materiale è individuale per se stessa. Poiché la natura è comune, si deve cercare in un altro principio

${ }^{74}$ Cf. L. Iammarrone, Giovani Duns Scoto. Metafisico e teologo. La tematiche fondamentali della sua filosofia e teologia, Roma 1999, ed. Miscellanea Francescana, p. 226.

75 Cf. G. Duns Scoto, Ordinatio, II, d. 3, p. 1, q. 1. 
che, nel caso di un ente individuale e concreto, la renderà individuale. Questo principio deve essere qualcosa di interno dell'ente e anche qualcosa di positivo, siccome l'ente singolare è più perfetto di quello universale.

Egli respinge anche la teoria secondo la quale la quantità sarebbe il principio di individuazione, perché è soltanto uno degli accidenti, e siccome loro dipendono dalla sostanza certamente non possono essere il principio di individuazione. Il fatto che la quantità è scindibile ha indotto alcuni filosofi a pensare che è essa il principio di individuazione. Ciò sarebbe possibile se essa fosse individuo in sé: formaliter de se haec. Ma siccome essa non lo è, e siccome non possiede individualità propria, essa non può attribuire alla sostanza ciò che essa stessa non possiede. ${ }^{76}$ Infatti, non è l'accidente della quantità ciò che individualizza la materia, ma al contrario, ogni materia individualizzata possiede la propria quantità, e ciò è compiuto dalla forma. Questo si vede meglio in una persona specifica, per esempio Aristotele, che possiede la sua anima individuale, che non è diventata individuale per l'unione con un corpo particolare, piuttosto viceversa.

Tenendo sempre presente il fatto che ogni individuo possiede una natura ad un modo personale e intercomunicabile, Duns Scoto ritiene che allora l'individuo deve possedere anche qualcosaltro oltre alla natura, qualcosa di positivo che determina la sua natura, al fine di garantire la propria individualità. ${ }^{77}$ Difatti, questa natura collettiva è indifferente nei confronti di questo o quell'individuo, e quindi ha bisogno di un altro principio che la renderà numericamente una in un particolare ente e diversa dagli altri enti. ${ }^{78}$ Nelle ricerche ulteriori, Duns Scoto si serve della differenza fra l'unione minore (minor unitate), il che è l'unità della natura, e l'unione maggiore (unitate maiore), che chiama anche numerico ed è essa l'unità dell'individuo. ${ }^{79}$ Però, qui si pone un problema. Ovvero, se l'unità numerica è quella più grande e anche quella che non consente la moltitudine, ed è proprio questo che Duns Scoto intende per 'individuazione', cioè qualcosa che appartiene a un singolo individuo e nessun altro, come si spiega allora l'esistenza della moltitudine di individui. In questo caso è necessaria l'esistenza di quella unità minore, che è alla base di un elemento comune che si trova

76 Cf. L. Iammarrone, Giovani Duns Scoto, p. 231.

77 Cf. G. Duns Scoto, Ordinatio, II, d. 3, p. 1, q. 5.

78 Cf. G. Duns Scoto, Ordinatio, II, d. 3, p. 1, q. 1.: “...natura est indifferens ad unitatem singularitatis."

79 Cf. G. Duns Scoto, Il principio di individuazione, p. 5. 
nelle creature. Ciò significherebbe che è grazie all'unità minore che esiste la moltitudine degli individui. ${ }^{80}$

Cercando il principio di individuazione, Duns Scoto si chiede se esiste negli enti sensibili una realtà positiva (aliqua entitas positiva) dotato dal proprio essere e che differisce dalla forma di tale ente. ${ }^{81}$ Egli ritiene che la risposta deve essere positiva, perché non avrebbe senso parlare di un ente complesso, se non esistono realmente in lui almeno due elementi di cui è composto. Perciò, egli ritiene che la materia ha una sua esistenza reale. Dobbiamo notare che Duns Scoto qui confonde i due modi di esistenza degli enti complessi, ovvero la complessità metafisica e la complessità fisica. Il primo modo non presuppone che ogni „elemento" della unione ha il suo essere, anzi può averlo soltanto un elemento grazie all'unità dell'ente complesso. L'unione fisica presuppone l'esistenza di "elementi" che sono diversi, e che in realtà sono già una cosa individuale e concreta, e che successivamente insieme costituiscono un terzo ente reale.

Affinché sia possibile una modifica, dal punto $\mathrm{A}$ al punto $\mathrm{B}$, è necessario che vi sia un elemento comune non appartenente allo stesso tempo né al punto A né al punto B, ma che essi lo possiedono successivamente. Questo elemento negli enti sensibili è la materia. Tuttavia, per quanto riguarda la natura di questo elemento c'erano pareri diversi nel corso della storia. Alcuni dicono che la materia è tutto, e questi Duns Scoto li chiama i materialisti, altri invece riducono tutti gli enti variabili alla forma. Lui invece non si soddisfa con questo, perché a suo parere nessuno di loro spiega il cambiamento, e l'unica differenza tra loro è di natura verbale. ${ }^{82}$ Tuttavia, qualsiasi sia il modo in cui viene compresa la materia, la sua natura è quella della potenza, poiché il suo ruolo nell'ente complesso è di essere la potenza rispetto alla forma che riceverà. Duns Scoto conosce due tipi di potenza e questa distinzione gli aiuterà a determinare meglio la natura della materia. Un tipo di potenza è „soggettivo” e l’altro „obiettivo”. ${ }^{83}$

${ }^{80}$ Cf. G. Duns Scoto, Il principio di individuazione, p. 131-132, nota 6.

${ }^{81}$ Cf. G. Duns Scoto, Ordinatio, II, d. 12, q. 1, n. 1.

${ }^{82}$ Cf. G. Duns Scoto, Ordinatio, II, d. 12, q. 1, n. 3: „differunt opiniones istae solum in voce et non in re".

${ }^{83}$ Duns Scoto con la potenza obiettiva intende la forma finale che l'ente nel cambiamento può raggiungere. È, per esempio, quando si dice che una certa quantità di tela oggettivamente è in potenza l'abito. La potenza soggettiva è lo stesso soggetto che al momento del cambio è in relazione al compimento di questo cambiamento. (cf. É. Gilson, Giovanni Duns Scoto, Milano 2008, Ed. Jaca Book, p. 451). 
Ma cosè questo qualcosa che Duns Scoto riconosce come il principio di individuazione? Per rispondere a questa domanda, bisogna tener a mente la sua distinzione tra l'unità numerica e l'unità specifica. Anche se ci sono somiglianze tra loro, l'unità dell'individuo è superiore, perché l'unità numerica è l'unità nel senso più stretto. ${ }^{84}$ Secondo Duns Scoto, quando una specie si unisce con un genere crea con esso un insieme che rappresenta una particolare quiddità (quiditas), e nel caso dell'uomo si tratta di animal rationale. Quando si tratta di unità numerica di un ente concreto, non viene a formarsi una quiddità nuova, ma un individuo concreto a un diverso livello di realtà della quiddità.

Quindi, per Duns Scoto il principio di individuazione non è né materia né forma, neanche gli accidenti, né tutti insieme, ma qualcosa dentro dellente che gli darà la sua individualità e singolarità. Questa è una realtà positiva, quale dal di dentro determina l'ente per l'individualità, e per „questa realtà positiva si dice che sia di per sé la causa di individuazione, perché sotto la 'individuazione' considero l'indivisibilità." ${ }^{85}$ Perciò egli ritiene che soltanto 'entità individuale' può causare 'differenza individuale', e questa non è né materia, né forma, né unione di materia e forma, perché ognuno è 'natura' di per se e quindi esse non implicano nessuna sorta di individualità. Quando egli spiega come un uomo dà alla luce un altro uomo, uguale per la forma, ma comunque diverso dal primo, allora egli dice che le loro haecceitates sono diverso, ma l'unità minore della forma è uguale. ${ }^{86}$

Che cosè allora il principio di individuazione secondo Duns Scoto? Non è facile rispondere a questa domanda senza ambiguità. Per quanto emerge come risposta questo famoso neologismo haecceitas, che domina in quasi tutte le storie della filosofia medievale, va notato che stesso Duns Scoto lo ha usato molto raramente. ${ }^{87}$ M. Fumagalli Beonio Brocchieri e M. Parodi ritengono pure

\footnotetext{
${ }^{84}$ Cf. L. Iammarrone, Giovani Duns Scoto, p. 233.

${ }^{85}$ G. Duns Scoto, Ordinatio, II, d. 3, p. 1, q. 2.: “...et ilud positivum erit illud quoddicetur esse per se causa individuationis, quia per individuationem intelligoillam indivisibilitatem."

${ }^{86}$ Duns Scoto scrive in Reportata Parisiensia, II, d. 12, q. 5: „Haecceitas generantis et geniti sunt primo diversa."

${ }^{87}$ Questo è il punto in cui lui lo usa, Reportata Parisiensia, II, d. 12, q. 6: „Sic haec unitas minor de se est haec numero, non essentialiter, sed tantum denominative; sed haecceitas est numero haec essentialiter". Lo si trova in un altro punto nella sua opera Quaestiones super libros Metaphysicorum Aristotelis, lib. VII, q. 13: “...quia si nulla unitas reali naturae est minor haecceitate...”. Antonello D’Angelo, editore della edizione italiano-latina del testo di Duns Scoto legato al principio di individuazione, come molti altri studiosi del pensiero di
} 
che egli ha usato raramente questo termine, ovvero soltanto nel suo commento di Sentenze, più precisamente nel suo lavoro Reportata Parisiensia. ${ }^{88}$ Un parere simile ha anche J. Kraus che considerava dubbia l'autenticità di questo neologismo, anche se concorda sul fatto che è stato molto usato nella sua scuola. ${ }^{89}$ Siccome i suoi discepoli e seguaci lo utilizzavano molto spesso, è rimasto dominante nella letteratura. Un esperto della filosofia medievale, É. Gilson, basandosi sulla ricerca di J. Kraus, mette in dubbio che il termine haecceitas era autentico di Duns Scoto e non della sua scuola che lo ha seguito. D’altra parte, lui critica il valore di questo termine, perché ci espone al fatto che un ente è una „cosa”, ma non il principio che nel senso espresso dell'attualità determina qualsiasi ente reale nella sua singolarità. ${ }^{90}$

Se accettiamo la tesi secondo la quale il principio di individuazione è haecceitas oppure entitas singularis, oppure proprietas individualis, come Duns Scoto lo chiamerà altrove, allora il processo di individuazione avverrebbe in questo modo. Questo principio determina la materia generale nella materia dell'ente concreto che è numericamente uno, così come determina la forma generale d’ente nella forma degli enti singolari, e allo stesso modo si unisce all'unione di materia e forma per ottenere un'ente concreto individuale e sensibile. ${ }^{91}$ Allo stesso modo vi si arriva all'individuazione degli accidenti dell'ente, e anche all'anima umana individuale. Per spiegare meglio questo principio di individuazione, egli lo paragona alla differenza specifica (differentia specifica) e lo chiama differentia individualis. E così come la differenza specifica determina la specie in relazione al genere, così pure la differentia individualis determina l'individuo in relazione alla specie, con un'osservazione importante che la differenza tra specie e haecceitas

Duns Scoto, fa notare che questo è un punto raro per Duns Scoto, dove lui usa il termine haecceitas (cf. G. Duns Scoto, Il principio di individuazione, p. 132, nota 8). Il termine "haecceitas" è difficile da tradurre; forse il significato più vicino sarebbe questità (nel senso 'questa cosa' e non altra). Questo termine vuole mettere in evidenza il fatto che si tratta di questo particolare ente individuale, che anche se possiede una natura comune (natura communis), è diverso da tutte le altre creature, per la sua questità, vale a dire, essa è questa, e non qualche altra cosa.

${ }^{88}$ Cf. M. Fumagalli Beonio Brocchieri - M. Parodi, Povijest srednjovjekovne filozofije, p. 364.

${ }^{89}$ Cf. J. Kraus, Die Lehre des Johanes Duns Scotus O.M.F. von der „natura communis”, Fribourg 1927.

90 Cf. É. Gilson, Giovanni Duns Scoto, p. 483.

91 Cf. R. Meßner, Il principio di individuazione nella visione scotista, http://siba-ese. unisalento.it/index.php/idee/article/view/3483/2881 (20.12. 2013). 
non è reale, ma soltanto formale. ${ }^{92}$ Questo principio interno dell'ente egli chiama anche ultima realitas entis, ${ }^{93}$ oppure ultima actualitas formae. ${ }^{94}$ É. Gilson spiega che qua si tratta in primo luogo dell'individuazione della quiddità e non dell'individuazione per la quiddità. Oppure, come lui suggerisce, „forse si potrebbe dire senza tradire Duns Scoto che è un'individuazione della forma, ma non mediante la forma". ${ }^{95}$

Da quanto esposto, è ovvio che stesso Duns Scoto non era soddisfatto del fatto che il termine haecceitas esprime sia l'individualità che la distinzione di un ente dall'altro, e con questi altri termini ${ }^{96}$ ha voluto esprimere la perfezione posseduta dall'individualità di tale ente in relazione all'universalità della natura, il quale è espressa tramite l'attualità della sua forma. Quindi, possiamo concludere che lui riteneva il haecceitas come l'espressione finale della forma dell'ente. Come se cercava un principio metafisico più profonda che sarebbe stato l'atto di tutti gli atti, ciò che Tommaso ha trovato nel suo actus essendi, ma che Duns Scoto non ha riconosciuto, e quindi neanche ammesso.

\section{Conclusione}

Possiamo dire in conclusione che cè una differenza notevole nelle risposte offerte da Duns Scoto e Tommaso d'Aquino. Il principio che potrebbe essere la fonte di individuazione secondo Duns Scoto agisce non nell'ordine di essere, come è il caso di actus essendi di Tommaso, ma nellordine di essenza o quiddità. Come conseguenza, ciò potrebbe avere un vantaggio nel contesto della visione di realtà di Duns Scoto. Siccome per lui, l'ente concreto è più perfetto dell'ente generale, e in modo particolare l'ente individuale umano, ovvero la persona umana, questo principio gli permette di arrivare nel processo conoscitivo fino

92 Cf. R. Meßner, Il principio di individuazione nella visione scotista, http://siba-ese. unisalento.it/index.php/idee/article/view/3483/2881 (20.12. 2013).

93 Cf. G. Duns Scoto, Il principio di individuazione, p. 96; Ordinatio, II, d. 3, pars 1, q. 6.: „Non est igitur 'ista entitas' materia vel forma vel compositum, in quantum quodlibet istorum est 'natura' - sed est ultima realitas entis..."

94 Cf. É. Gilson, Giovanni Duns Scoto, p. 719; L. Iammarrone, Giovani Duns Scoto, p. 234.

95 É. Gilson, Giovanni Duns Scoto, p. 483.

96 Riportiamo ancora una volta alcuni termini usato da Duns Scoto, e che dovrebbero sostituire il neologismo haecceitas nel suo significato. Questi sono: ultima realitas entis; ultima actualitas formae; differentia individualis; entitas singularis i proprietas individualis. 
alla persona umana individuale. Ciò significa che nel processo conoscitivo non ci fermiamo a livello di specie, ma continuiamo fino alla conoscenza della singola persona umana. ${ }^{97} \mathrm{R}$. Meßner ritiene che la scoperta di haecceitas ha portato Duns Scoto, nel senso cognitivo, molto avanti, in modo che ha insegnato che la conoscenza delle cose individuali è la più perfetta forma della conoscenza, poiché in essa, oltre alle caratteristiche individuali delle cose individuali, sono incluse anche le caratteristiche del genere e della specie. In questo modo, egli ha aperto la strada per la conoscenza della persona umana individuale, il che ha un valore inestimabile per i problemi contemporanei. ${ }^{98}$

Tuttavia, vorremo qui sottolineare il fatto che nella definizione del principio di individualizzazione di Tommaso non cè la materia, ma la materia quantitate signata. Cioè, la materia determinata dalla forma, il che significa che la materia è trasportata dalla potenza in attualità, e questa attualizzazione è compiuta dalla forma. È chiara la dottrina di san Tommaso che la forma porta l'essere all'ente, e questo in definitiva è grazie all'atto di tutti gli atti o come dice san Tommaso actus essendi. Questo è, possiamo concludere, il principio più profondo, non solo di attualità, ma anche individualità dell'ente reale, della sua materia e forma e anche unione di materia e forma, e anche di tutti gli accidenti del singolo ente concreto.

Se le cose sono così, e noi crediamo di si, allora va detto che in questo punto non cè molta differenza tra Tommaso d'Aquino e Duns Scoto. Perché che cosè, alla fine, la differentia individualis di Duns Scoto, che dovrebbe essere per se stessa all'interno della sostanza concreta, che le dà la quiddità ed essere? Non è nient'altro che l'atto di quiddità. ${ }^{99}$ E questo significa nella costituzione metafisica degli enti per Duns Scoto haecceitas ha lo stesso ruolo come actus essendi per Tommaso d'Aquino. E quindi possiamo concordare con l'affermazione di É. Gilson che „nel cuore della realtà, in Tommaso d'Aquino, si trova l'atto di essere; in Duns Scoto, l'ecceità". ${ }^{100}$

Il principio di individuazione che abbiamo cercato è una cosa più intima dell'ente, perché necessariamente fa che io sono io, e non tu, e quindi deve

${ }^{97}$ Infatti, Duns Scoto nel suo commento di Metafisica di Aristotelove Metafizike scrive che l'individuo è l'ente più vero (cf. Quaestiones super libros VII Metaphysicorum Aristotelis, q. 13: "Individuum est verissime ens..."

98 Cf. R. Meßner, Il principio di individuazione nella visione scotista, p. 34-36.

99 Cf. G. Duns Scoto, Reportata Parisiensia, II, d. 12, q. 8.: „Sed si esset res alia, proprie esset actus quidditatis."

${ }^{100}$ É. Gilson, Giovanni Duns Scoto, p. 485. 
essere qualcosa che non mi è esterno, ma qualcosa che mi appartiene e che mi rende profondamente determinato dall'interno. Allo stesso tempo, ciò deve essere quello che fa che io sono. Ciò che fa che io sono non è quello che fa che tu sei. Perciò deve essere qualcosa collegato con la forma, ma in modo tale che sia mia e non tua forma, nonostante sia la mia che la tua forma sono umane. Ciò deve essere qualcosa di più profondo della forma stessa, pur appartenendo alla forma. Noi riteniamo che questo è l'originale actus essendi di Tommaso, che lui chiama l'atto di tutti gli atti, e noi lo chiameremmo anche il principio di tutti i principi.

\section{Bibliography}

Abelardo P., Logica ingredientibus, http://www.forumromanum.org/literature/abelardx. html (05.12. 2015).

Aristotele, Fisica, Roma 2005, ed. Laterza.

Aristotele, Metafisica, 3 vol., Milano 1993, ed. Vita e Pensiero.

Aristotele, Dell'Anima, Roma 2007, ed. Laterza.

Artigas M., Sanguineti J. J., Filosofia della natura, Firenze 1989, ed. Le Monnier.

Boethius, De Trinitate, trad. ital.: S. Boezio, La consolazione della filosofia. Gli opuscoli tologici, Milano 1979, ed. Rusconi.

Bonaventura, Commentaria in IV libros Sententiarum, Quaracchi 1882-1902.

Duns Scoto G., Il principio di individuazione. Ordinatio II, d. 3, pars 1, Quaestiones 1-7, Napoli 2011, ed. Il Mulino.

Duns Scotus I., Opera omnia, Editio minor, Alberobello 1998-2001, Editrice AGA.

Fumagalli Beonio Brocchieri M., Parodi M., Povijest srednjovjekovne filozofije, [Storia della filosofia medievale, Roma 2005, ed. Laterza], Zagreb 2013, ed. Kršćanska sadašnjost.

Gilson É., La filosofia di San Bonaventura, Milano 1995, ed. Jaca Book.

Gilson É., Giovanni Duns Scoto, Milano 2008, ed. Jaca Book.

Gracia J. J. E., (a cura di), Individuation in Scholasticism: The Later Middle Ages and the Counter-Reformation (1150-1650), Albany 1994, ed. State University of New York.

Gracia J. J. E., Introduction to the problem of individuation in the early Middle Ages, München - Wien 1984, ed. Philosophia Verlag.

Grenet P.-B., Ontologia, Brescia 1967, ed. Paideia.

Iammarrone L., Giovani Duns Scoto. Metafisico e teologo. La tematiche fondamentali della sua filosofia e teologia, Roma 1999, ed. Miscellanea Francescana.

King P., The Problem of Individuation in the Middle Ages, „Theoria” 66 (2000), p. 159-184; http://individual.utoronto.ca/pking/articles/Mediaeval_Individuation.pdf(07.12.2013). 
Kraus J., Die Lehre des Johanes Duns Scotus O.M.F. von der „natura communis”, Fribourg 1927.

Meßner R., Il principio di individuazione nella visione scotista, „Idee” 64 (2007), p. 19-41.

Mondin B., La metafisica di S. Tommaso d'Aquino e i suoi interpreti, Bologna 2002, Edizioni Studio Domenicano.

Owens J., The doctrine of being in the Aristotelian Metaphysics, Toronto 1978, ed. Pontificial Institute of Medieval Studies.

Reale G., Per una nuova interpretazione di Platone. Riletura della matafisica dei grandi dialoghi alla luce della „Dotrine non scritte”, Milano 1993, ed. Vita e Pensiero.

Reale G., Storia della filosofia antica, II: Platone e Aristotele, Milano 1997, ed. Vita e Pensiero.

Reale G., Storia della filosofia antica, V: Lesico indici e bibliografia, Milano 1997, ed. Vita e Pensero.

Regis E., Aristotle’s ‘principle of individuation', „Phronesis” 21 (1976), p. 157-166.

Ross D., Aristotle, London 1995, Routlidge.

Tommaso d'Aquino, Opera omnia, http://www.corpusthomisticum.org/ (05.12. 2015). 